JOURNAL OF

APPLIED

CRYSTALLOGRAPHY

ISSN 1600-5767

Received 4 April 2017

Accepted 4 April 2017

Keywords: crystal forms; systematic absences; Miller indices; symmetry restrictions; crystallographic education.

\section{The ash heap of crystallography: restoring forgotten basic knowledge. Corrigendum}

\author{
Massimo Nespolo* \\ Université de Lorraine, CRM2, UMR 7036, Vandoeuvre-les-Nancy, F-54506, France, and CNRS, CRM2, UMR 7036, \\ Vandoeuvre-les-Nancy, F-54506, France. *Correspondence e-mail: massimo.nespolo@univ-lorraine.fr
}

One imprecise and one incomplete statement in the article by Nespolo [J. Appl. Cryst. (2015), 48, 1290-1298] are corrected.

In our article (Nespolo, 2015), one imprecise and one incomplete statement about the conditions for Miller indices to be relatively prime have been identified, which we correct hereafter.

Consider the statement on page 1293, after equation (2): 'When the unit cell is primitive or when the planes of the $(h k l)$ family are parallel to the face of the unit cell containing the centring vector, the above condition is consistent with coprime Miller integers.' In that sentence, the term 'the centring vector' should be replaced by 'a centring vector'. In the example given for the $o C$ unit cell in Fig. 1 therein, centring vectors go from the origin to the lattice nodes $\frac{1}{2} \frac{1}{2} 0, \frac{1}{2} \frac{1}{2} 0, \frac{1}{2} \frac{1}{2} 0$ and $\frac{1}{2} \frac{1}{2} 0$. These are all contained in the (001) plane, but only two of them, namely $\frac{1}{2} \frac{1}{2} 0$ and $\frac{1}{2} \frac{1}{2} 0$, are contained in the (110) plane.

On the same page, equation (2),

$$
h x+k y+l z=n,
$$

is followed by the statement 'it should give the first plane of the family after the origin, in the positive direction, when $n=$ 1 ', which should be completed by the condition 'and $n$ is minimal'. For example, in the case of the plane (301), all the

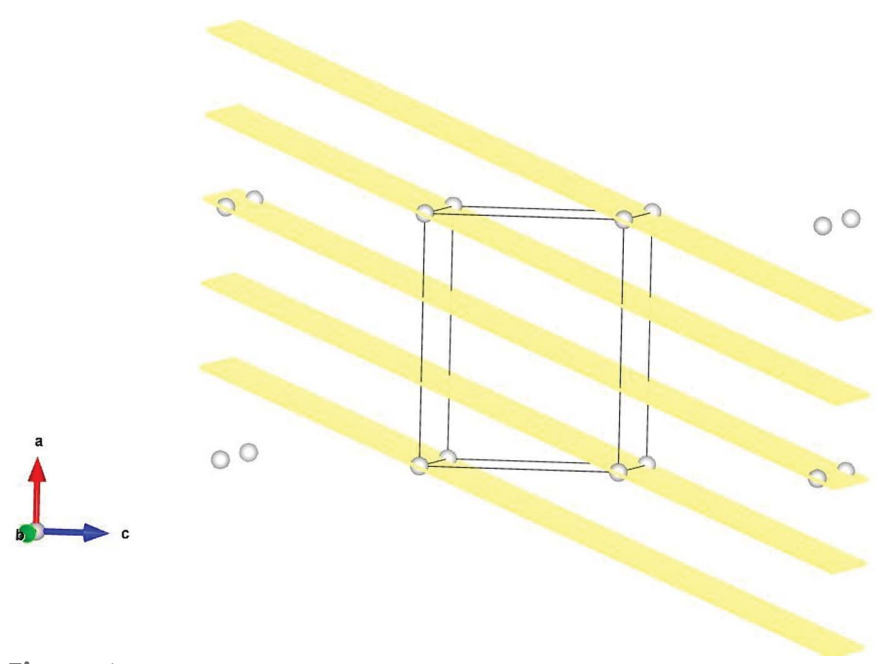

Figure 1

The (301) family of lattice planes for a primitive unit cell. From bottom to top of the figure, the planes correspond to $n=0,1,2,3,4$ in equation (2). 


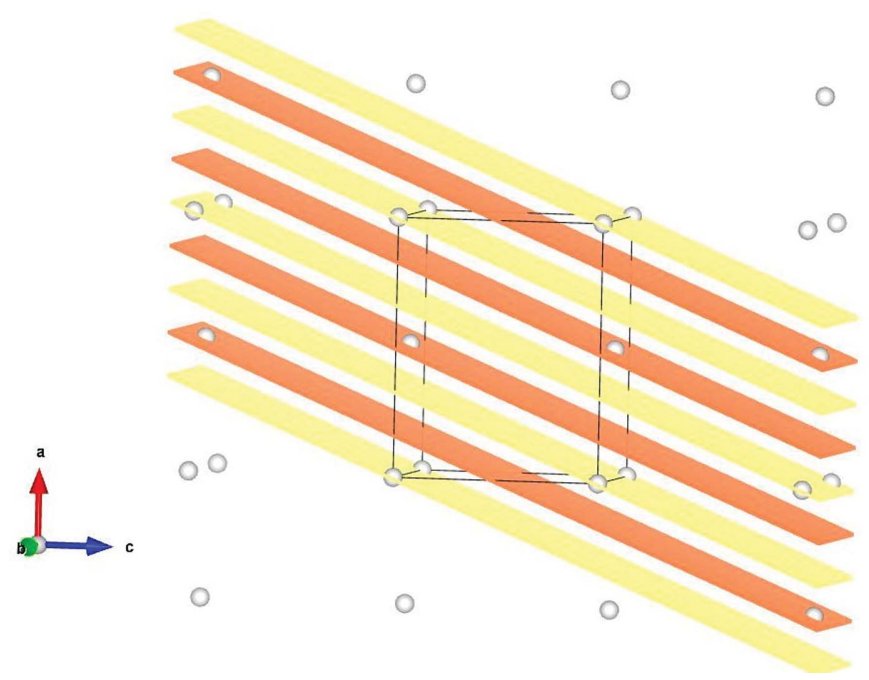

Figure 2

The same family of lattice planes for a $C$-centred unit cell. Planes shown in orange do not exist in a primitive unit cell. If the same Miller indices, (301), are used, then these planes would correspond to half-integer values of $n$ in equation (2) and the one leading to $n=1$ is not the first plane of the family but the second one, contrary to the definition of Miller indices. Accordingly, from bottom to top of the figure, the planes would correspond to $n=0, \frac{1}{2}, 1, \frac{3}{2}, 2, \frac{5}{2}, 3, \frac{7}{2}, 4$ in equation (2). By adopting the correct non-relatively prime indices (602), the first plane of the family does correspond again to $n=1$ in equation (2); from bottom to top of the figure, the planes correspond now to $n=0,1,2,3,4,5,6,7,8$. Figures drawn with VESTA (Momma \& Izumi, 2011). planes of the family are parallel to the $b$ axis. If the unit cell is primitive, the three first planes have intercepts on the $a$ and $c$ axes at $x=\frac{1}{3}, z=1 ; x=\frac{2}{3}$ and $z=2$; and $x=1$ and $z=3$, respectively (Fig. 1). Accordingly, they pass through lattice nodes $0 v 1,0 v 2$ and $1 v 0$ (any integer $v$ ), which leads to $n=1,2$ and $n=3$ in equation (2). In this case, (301) are the correct Miller indices. If the unit cell is $C$ centred, however, the first three planes for the family have intercepts $x=\frac{1}{6}, z=\frac{1}{2} ; x=\frac{1}{3}$, $z=1 ; x=\frac{1}{2}, z=\frac{3}{2}$ (Fig. 2). Accordingly, they pass through lattice nodes $\frac{1}{2}\left(v+\frac{1}{2}\right) 1,0 v 1$ and $\frac{1}{2}\left(v+\frac{1}{2}\right) 0$ (any integer $v$ ), which leads to $n=0.5,1$ and $n=1.5$, respectively. Because the minimal value of $n$ is not 1, the Miller indices of the family are not (301) but (602).

\section{Acknowledgements}

We thank Professor Carolyn P. Brock (University of Kentucky, USA) for pointing out the incorrect statements.

\section{References}

Momma, K. \& Izumi, F. (2011). J. Appl. Cryst. 44, 1272-1276. Nespolo, M. (2015). J. Appl. Cryst. 48, 1290-1298. 